\title{
Optimization of a headspace solid-phase microextraction method for the gas chromatography-mass spectrometry analysis aroma compounds of Litsea mollis Hemsl. immature fruit
}

\author{
Yi-Ni YANG ${ }^{1,2}$, Miao LIANG ${ }^{2}$, Yan YANG ${ }^{2}$, Fu-Ping ZHENG ${ }^{1}$, Xing-Ping WANG ${ }^{3}$, Ai-Nong YU ${ }^{1,2 *}$
}

\begin{abstract}
This study optimized using a headspace solid-phase microextraction (HS-SPME) method for extracting volatile aroma compounds, terpenoids in particular, from Litsea mollis Hemsl. immature fruit (LMIF). Gas chromatography-mass spectrometry was used to separate and identify volatile terpenoids in LMIF. The types of SPME fiber coating, salt addition, and desorption time were optimized by single factor-experiments to determine the range of extraction temperature. Built on insights from the single factor-experiments, the response surface methodology was further used to optimize the extraction temperature, extraction time, and equilibrium time of HS-SPME. The results showed that the optimal HS-SPME conditions for extracting volatile terpenoids from LMIF were: $50 / 30 \mu \mathrm{m}$ of DVB/CAR/PDMS SPME fiber, $2.0 \mathrm{~g}$ of added sodium chloride, desorption at a temperature of $250{ }^{\circ} \mathrm{C}$ for $3 \mathrm{~min}$, extraction and equilibrium temperature of $46^{\circ} \mathrm{C}$, extraction time of $36 \mathrm{~min}$, and equilibrium time of $20 \mathrm{~min}$. Under the above conditions, the predicted value of extraction was 66.92 , while the experimental value was 65.78 . The prediction value matched well with the experimental value with good repeatability. The designed model was proven to be valid, which can be applied for future extraction of aroma compounds from IMIF.
\end{abstract}

Keywords: Litsea mollis Hemsl.; solid-phase microextraction; response surface methodology; terpenoid; citral; aroma compound.

Practical Application: The study provides valuable and reliable information that volatiles extraction is an efficient and environment-friendly process for extracting aroma compounds from LMIF, and will help in the development of a natural food for food industries.

\section{Introduction}

Litsea mollis Hemsl. (LM) is a plant of Lauraceae. In China, it is mainly distributed in Guangdong, Guangxi, Hunan, Hubei, Sichuan, Guizhou, Yunnan, and eastern Tibet (Chinese Academy of Sciences, 2019). Its fruit and flower are rich in volatile oil, which is an aromatic yellowish oily liquid. The volatile oil has antibacterial activities (Xiao \& Li, 2007) and also is antitussive and expectorant (Zhou, 1995). In southwestern China, LM immature fruit (LMIF) is widely used as a spice. LMIF can be made into popular appetizing cold dishes by pickling with soy sauce, vinegar, pepper, garlic, and ginger (Zhang, 2015).

As a spice or appetizing cold dish, the aroma of LMIF is a very important aspect of quality. However, the composition of the aroma of edible LMIF is yet elusive. Studies have shown that the main chemical components of LM fruit volatile oil extracted by steam distillation are terpenoids (more than $80 \%$ ), including $\alpha$-citral (39.19\%) and $\beta$-citral (30.19\%) (Chen et al., 1984). However, these data do not reveal their original aroma components, as volatile compounds might undergo hydrolysis and thermal decomposition during the distillation process.

In order to identify the aroma components of LMIF, we used a headspace solid-phase microextraction (HS-SPME) method

to extract aroma compounds from IMIF, which is usually used as an appetizing cold dish. We further optimized the extraction parameters and enriched the aroma components of this juice. The extraction and enrichment of aroma compounds with HS-SPME are affected by a variety of factors including types of SPME fiber, amount of salt added to the extract, desorption time, extraction temperature and time, and equilibrium temperature and time (Vichi et al., 2006). In this work, we conducted single factor-experiments to optimize the type of SPME fiber coating, salt conditions, and range of extraction temperatures. Furthermore, built on insights from the single factor-experiments, this study further used a response surface methodology (RSM) to optimize the extraction temperature, extraction time, and equilibrium time of HS-SPME. RSM is the most relevant technique used for variable optimizations (Martins et al., 2013), using the Box-Behnken experimental design with multiple quadratic regression to fit functional relationships between factors and response values for determining the optimal parameters (Bezerra et al., 2008). The main objectives of this study were to determine the significant process variables as well as the optimal conditions of HS-SPME that would allow for the maximum extraction of aroma compounds from LMIF. 
Our results provide a scientifically sound method for the study of volatile aroma components of LMIF.

\section{Materials and methods}

\subsection{Plant material}

Wild LMIFs with similar sizes were manually collected from healthy plants grown on field hillsides in Maoba Village, Sancha Township, Enshi, Hubei Province, China $\left(30^{\circ} 18^{\prime} \mathrm{N}, 109^{\circ} 34^{\prime} \mathrm{E}\right.$, $600 \mathrm{~m}$ above sea level) in May 2018. In order to prevent possible enzymatic reactions that can lead to the conversion of volatile compounds to their derivatives (Klesk et al., 2004), LMIFs after collection were immediately transported to a laboratory and stored in refrigerated boxes at $-78^{\circ} \mathrm{C}$. The sizes of LMIFs were measured to be $4-4.5 \mathrm{~mm}$. The fruits were identified and validated by Prof. Yong-Mei Yi, School of Forestry and Horticulture, Hubei University for Nationalities.

\subsection{Chemicals}

C5-C22 $n$-alkanes, eucalyptol (99.0\%), linalool (97.0\%), a-terpineol (95.0\%), geraniol (97.0\%), citral (98.0\%, $\beta$-citral: $\alpha$-citral $=1: 1)$, and 4-methyl-2-pentanol (98.0\%) were purchased from Sigma-Aldrich Chemical Co. (St. Louis, MO, USA). Sodium chloride was obtained from Sinopharm Chemical Reagent Co., Ltd. (analytical grade, Beijing, China). Ethanol was obtained from Shanghai Aladdin Bio-Chem Technology Co., LTD (Shanghai, China). Double-distilled water was used in all experiments.

\subsection{LM juice preparation and single factor-experiments}

$500 \mathrm{~g}$ of fruit was thawed and immediately juiced with a JYZ-E16 Juicer (Joyoung Co., Ltd., China). The obtained juice was immediately centrifuged at $8,000 \mathrm{rpm}$ for $20 \mathrm{~min}$ at $4{ }^{\circ} \mathrm{C}$ (Avanti J-30I Centrifuge, Beckman Coulter Inc., Brea, CA, US), and the supernatant (juice) was collected with a volume of $210.0 \mathrm{~mL} .10 \mathrm{~mL}$ of supernatant was mixed with $20.0 \mu \mathrm{L}$ of 4 -methyl-2-pentanol $(0.9155 \mathrm{~g} / \mathrm{L}$ in ethanol) as an internal standard. The mixture was put in a $20-\mathrm{mL}$ headspace extractor along with a magnetic stirrer and then sealed with a PTFE-silicon septum. In order to optimize the HS-SPME extraction of volatiles from LMIFs, we chose four factors for conducting single factor-experiments. These four factors were types of stationary phase of the SPME fiber, amount of salt added in the juice, desorption time, and extraction temperature. Further, we used RSM to optimize the most important parameters: extraction temperature and time, and equilibrium time of the HS-SPME procedure. For each sample of LMIF juice, three independent extractions were conducted.

\subsection{RSM experimental design and statistical analysis}

RSM was employed to optimize the most important parameters of the SPME procedure for the extraction of aroma components including terpenoids from LMIFs. The effects of three independent variables, including extraction temperature $\left(\mathrm{X}_{1}\right)$, extraction time $\left(\mathrm{X}_{2}\right)$, and equilibrium time $\left(\mathrm{X}_{3}\right)$ on the extraction yields of terpenoids were investigated using Box-Behnken experimental design. Based on single factor-experiments (data only shown for temperature selection), the range of $\mathrm{X}_{1}$ was $30-50^{\circ} \mathrm{C}, \mathrm{X}_{2}$ was 20-50 min, and $X_{3}$ was 10-30 min. The experimental design was summarized in Table 1. Also, types of stationary phase of SPME fiber, amount of salt, and desorption time were optimized using single factor experiments.

In order to model linear and quadratic effects of these factors, the Box-Behnken design (BBD) was implemented using 17 triplicated extractions of fully randomized experimental points. Further, five replicates of the center point were used to evaluate the pure error. The experimental data were analyzed using a quadratic stepwise regression. A second-order polynomial as shown in Equation 1 was used to express responses as a function of the independent variables:

$Y=\beta_{0}+\sum_{i=1}^{3} B_{i} X_{i}+\sum_{i=1}^{3} B_{i i} X_{i}^{2}+\sum_{i=1}^{3} B_{i j} X_{i} X_{j}+\varepsilon$

where $Y$ is measured response (concentration); $\mathrm{X}_{1}, \mathrm{X}_{2}, \mathrm{X}_{3}$ are the independent variables; $\beta_{0}, B_{i}, B_{i j}, B_{i j}$ are the regression coefficients of variables for intercept, linear, quadratic, and interaction terms, respectively. The fitness of the quadratic polynomial model was evaluated using model analyses, lack-of-fit tests, and coefficients of determination $\left(\mathrm{R}^{2}\right)$ as outlined by previous papers (Chen et al., 2012; Ma et al., 2013). The test of statistical significance was based on the total error criteria with a confidence level of $95.0 \%(p<0.05)$.

The experimental design and data analysis for multivariate optimization of HS-SPME procedure were performed using the Design-Expert 10 Trial software (State-Ease Inc., Minneapolis, MN, USA). The mean, standard deviation, and one-way analysis of variance (ANOVA) followed by Tukey's post hoc test were performed to compare differences in the mean of terpenoid contents of LMIFs. Differences were considered statistically significant at the $p$-value less than 0.05 .

\subsection{GC-MS analysis}

The analysis was conducted with a Concept Multifunctional System headspace autosampler (PAS Technology, Magdala, Germany) connected to an Agilent 6890N gas chromatograph (GC) coupled with an Agilent 5975i mass spectrometry (MS) selective detector (Agilent, Santa Clara, CA). Based on a previously report (Yang et al., 2019), an HP-5MS capillary column $(30 \mathrm{~m} \times 0.25 \mathrm{~mm}$ i.d $\times 0.25 \mu \mathrm{m})$ was used to separate the volatile compounds. Helium at a flow rate of $0.9 \mathrm{~mL} / \mathrm{min}$ was used as the carrier gas. The temperatures of injector, ion source, and interface were set to $250^{\circ} \mathrm{C}, 230^{\circ} \mathrm{C}$, and $280^{\circ} \mathrm{C}$, respectively. The oven temperature was programmed as follows: $40^{\circ} \mathrm{C}$ (held for $4 \mathrm{~min}$ ) to $260^{\circ} \mathrm{C}$ at an increment of $5^{\circ} \mathrm{C} / \mathrm{min}$, and then to $280{ }^{\circ} \mathrm{C}$ at an increment of $15{ }^{\circ} \mathrm{C} / \mathrm{min}$ (held for

Table 1. Factors and their coded values used for optimization.

\begin{tabular}{lccc}
\hline \multicolumn{1}{c}{ Factor } & \multicolumn{3}{c}{ Level } \\
\cline { 2 - 4 } & -1 & 0 & 1 \\
\hline Extraction temperature $\left({ }^{\circ} \mathrm{C}\right), \mathrm{X}_{1}$ & 30 & 40 & 50 \\
Extraction time $(\mathrm{min}), \mathrm{X}_{2}$ & 20 & 35 & 50 \\
Equilibrium time $(\mathrm{min}), \mathrm{X}_{3}$ & 10 & 20 & 30 \\
\hline
\end{tabular}


$1 \mathrm{~min})$. The MS spectra were obtained with a mass selective detector operated at an electron impact of $70 \mathrm{eV}$ in an $\mathrm{m} / z$ range of 20-400 amu at a scan rate of $1 \mathrm{scan} / \mathrm{s}$. To determine the peak area of each compound, the selected ion monitoring (SIM) peaks were extracted from the full scan chromatograms. The linear retention indices (LRI) of the analytes were calculated with the retention time of $\mathrm{C} 5-\mathrm{C} 22 n$-alkanes obtained under the same conditions. Volatile compounds were identified by comparing their MS data with those in the Nist08 and Wiley275 libraries and by comparing their LRIs in the National Institute of Standards and Technology (NIST) Gas Chromatography Library (National Institute of Standards and Technology, 2019), and with authentic samples. Each test was repeated twice to ensure the accuracy of qualitative analysis.

\section{Results and discussion}

\subsection{Single factor-experiments for the optimization of extraction conditions}

Using our previous extraction conditions (Yang et al., 2019), we conducted preliminary studies with a DVB/CAR/PDMS SPME fiber. The extraction and equilibrium temperature was set at $50{ }^{\circ} \mathrm{C}$ for 30 minutes, desorption time was set for 3 minutes, and the amount of salt was 2 grams. The total ion chromatogram of LMIF volatiles is shown in Figure 1. The results showed that the main volatiles are terpenoids, of which eucalyptol, linalool, $\alpha$-terpineol, $\beta$-citral, geraniol, and $\alpha$-citral were the main components. Subsequently, the peaks of theses six main compounds in the total ion chromatogram of volatile were selected to calculate the peak areas as an evaluation index for optimizing extraction conditions.

\section{Selection of SPME fiber coatings}

The coating material of HS-SPME fiber affects the adsorption sensitivity and selectivity. Under static conditions, the polarity and film thickness of the adsorbing fibers are the key factors affecting the adsorption efficiency. In order to select the best stationary phase, three SPME fiber coatings were tested, including PMDS (100 $\mu \mathrm{m}$; Supelco, Bellefonte, PA), CAR/PDMS ( $85 \mu \mathrm{m}$; Supelco, Bellefonte, PA), and DVB/CAR/PDMS SPME Fiber (50/30 $\mu \mathrm{m}$; Supelco, Bellefonte, PA). During testing, all of the fibers had the same length at $1 \mathrm{~cm}$ to offer better comparison. The extraction conditions were as follows: equilibrium time of $10 \mathrm{~min}$, extraction time of $30 \mathrm{~min}$, temperature of $40^{\circ} \mathrm{C}$, and salt amount of 8 grams.

The results of comparing different fiber coatings are shown in Figure 2A. Based on these data, it can be seen that the mixed fiber coatings DVB/CAR/PDMS was the most efficient to extract terpenoids other than $\alpha$-citral. Especially, the extraction efficiency of DVB/CAR/PDMS fiber for extracting eucalyptol, linalool, a-terpineol, and $\beta$-citral was significantly higher than that of the other two SPME fibers. Higher levels of a-citral could be extracted using PDMS fibers, but other compounds extracted using PDMS fibers are less efficient. Based on these results, combined with our previous reported results (Yang et al., 2019)

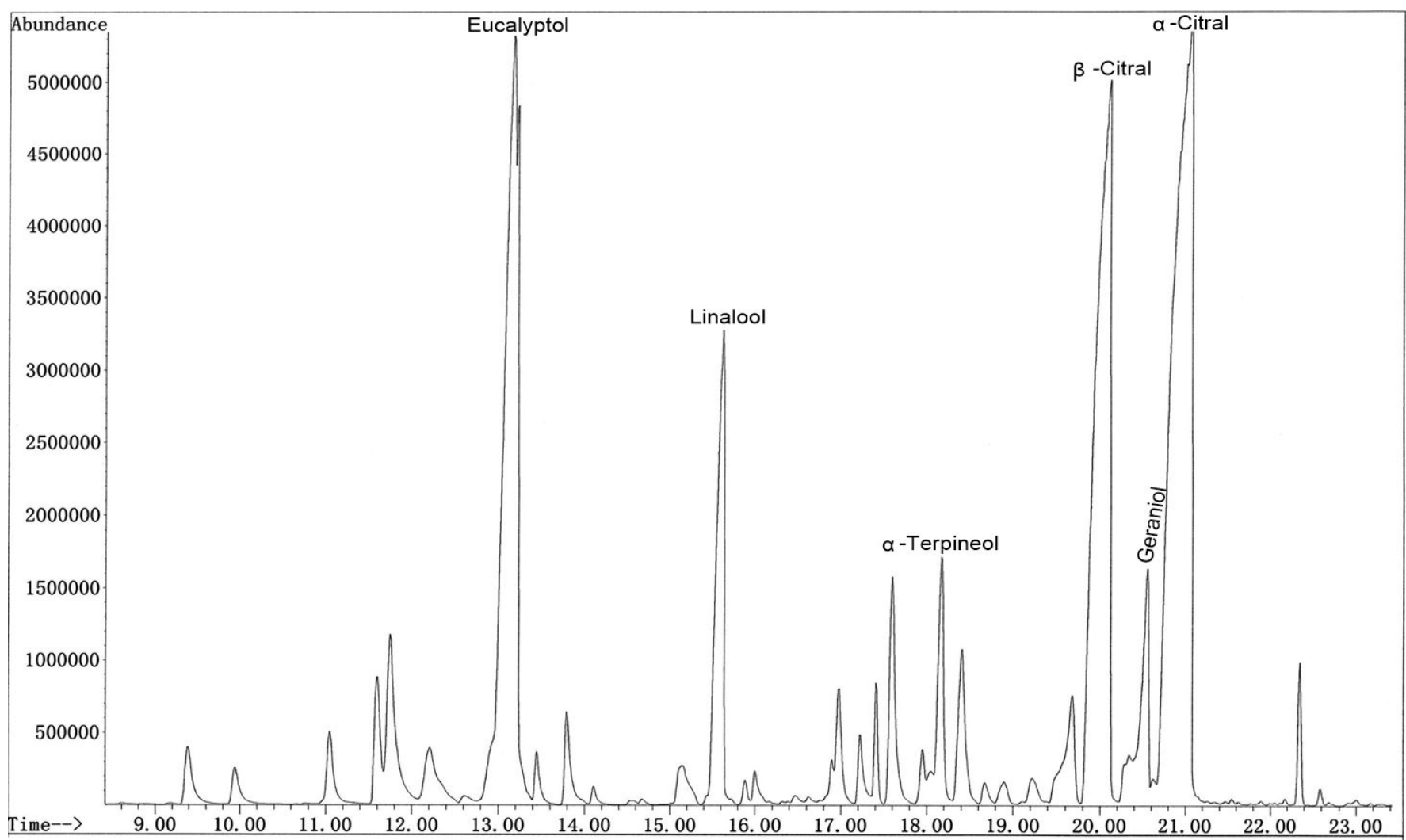

Figure 1. Total ion chromatogram of volatile compounds in LMIF using a DVB/CAR/PDMS SPME fiber. Extraction conditions: extraction and equilibrium temperature $50^{\circ} \mathrm{C}$, extraction and equilibrium time $30 \mathrm{~min}$, desorption time $3 \mathrm{~min}$, and salt addition $2 \mathrm{~g}$. 
and literature reports (Chmiel et al., 2017), we determined that the fiber coated with the DVB/CAR/PDMS coating was the most suitable for extracting main volatiles in LMIF.

\section{Effect of salt addition}

In general, adding an inorganic salt in a solution leads to increased ionic strength which consequently reduces the solubility of organic compounds (e.g. terpenoids) in the sample matrix and hence accelerates their release into the headspace. Thus, adding salts promotes concentrating analytes, particularly polar volatile compounds, in the sample headspace, and is able to improve the efficiency and sensitivity of the HS-SPME extraction (Peña et al., 2005).

The effect of the salt addition on the extraction efficiency of terpenoids was studied using different amounts of $\mathrm{NaCl}$ ranging from 0 to 3 grams. Figure 2B shows the impact of salting-out effect of $\mathrm{NaCl}$ on analytes after being absorbed by the SPME fiber. The results show that a high extraction efficiency of sterol and furfural was achieved when adding $2 \mathrm{~g}$ of $\mathrm{NaCl}$. The extraction efficiency of $\alpha$-citral was the highest when adding $1 \mathrm{~g}$ of $\mathrm{NaCl}$, but it was not significant higher compared with adding $2 \mathrm{~g}$ of $\mathrm{NaCl}$.
When the amount of $\mathrm{NaCl}$ was increased to $3 \mathrm{~g}$, the extraction efficiency did not further increase compared with adding $2 \mathrm{~g}$ of $\mathrm{NaCl}$. Only the yield of eucalyptol was relatively higher, the extraction efficiency of other compounds was not improved. The salting-out effect depends on the structure and chemistry of the analytical compound, especially the polarity. Based on these data, it could be inferred that the increased ionic strength has a positive effect on the extraction efficiency of terpenoids. However, excess amount of salt does not further increase the extraction efficiency. Using $2 \mathrm{~g}$ of $\mathrm{NaCl}$ is suitable for efficiently and cost-effectively extracting aroma compounds from LMIF.

\section{Effect of desorption temperature and time}

Desorption temperature and time are important factors affecting the transfer of analytes from fiber coatings to the chromatography system. They should be set to allow for complete desorption of analytes from the SPME fiber, but avoid thermal degradation of analytes and fiber coatings. At optimal desorption conditions, the carryover will be minimized and hence improves peak shapes. During a previous study on volatile profile of Rubus corchorifolius fruits, a desorption temperature of $250{ }^{\circ} \mathrm{C}$
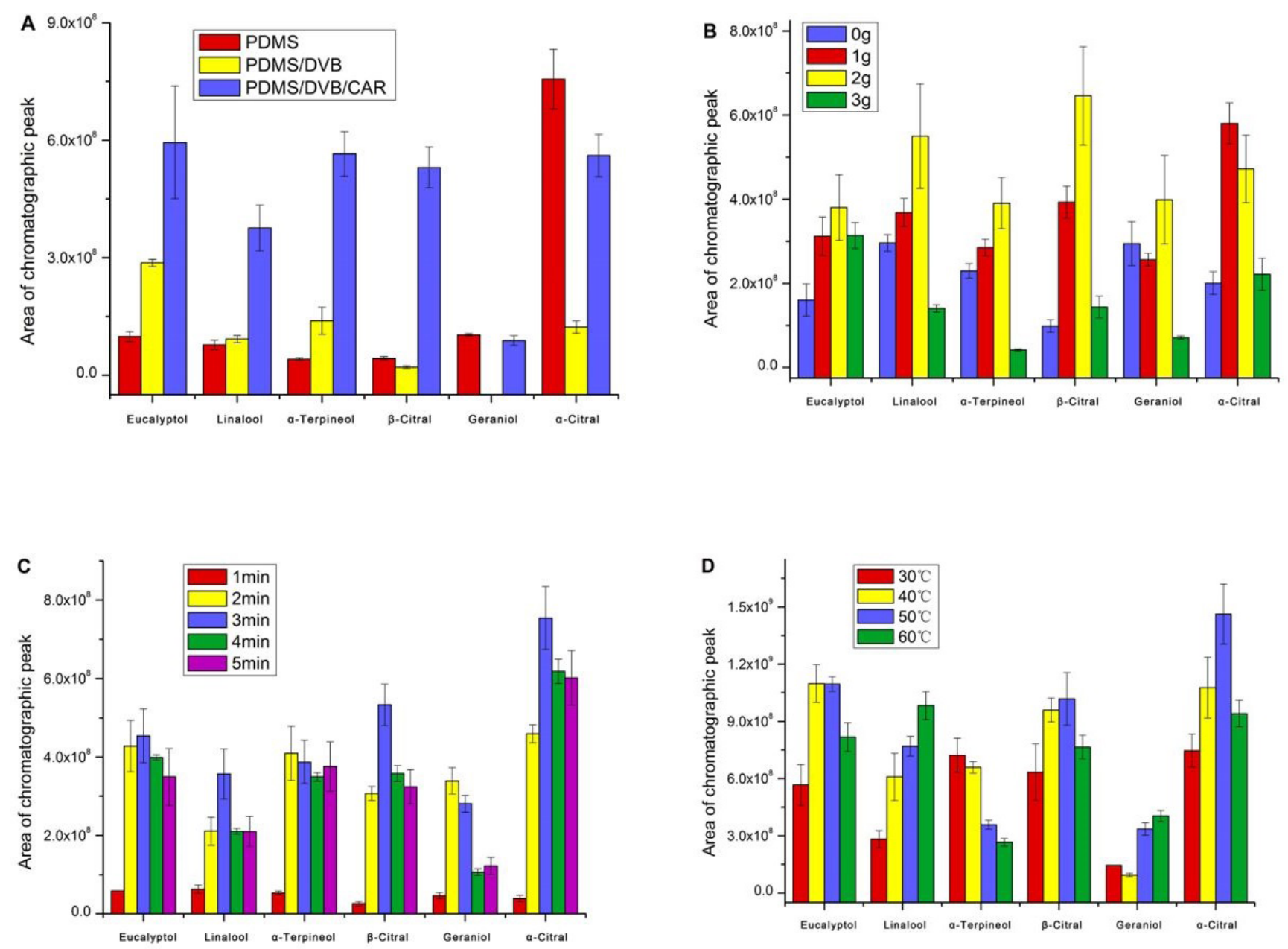

Figure 2. Extraction optimization by single factor-experiments: (A) types of SPME fiber coating; (B) salt addition; (C) desorption time; and (D) extraction temperature. The error bars represent the standard deviation of triplicate data. 
was used (Yang et al., 2019). Considering literature data on the extraction of erpenes and terpenoids from grape samples using the HS-SPME technique (Rocha et al., 2007), as well as the manufacturer's recommended temperature for DVB/CAR/PDMS fiber $\left(230-270^{\circ} \mathrm{C}\right)$, desorption temperature of $250^{\circ} \mathrm{C}$ was found to be optimal. Figure $2 \mathrm{C}$ shows the results of analytes obtained from varied desorption times. The most intense chromatographic peaks for most of the studied terpenoids were recorded after $3 \mathrm{~min}$ of desorption. Therefore, desorption at $250{ }^{\circ} \mathrm{C}$ for $3 \mathrm{~min}$ was chosen as the optimal condition for further studies.

\section{Effect of extraction temperature}

The extraction temperatures strongly affect the HS-SPME technique as the partition coefficients are temperature-dependent. In addition, the extraction temperature is closely related to the extraction time, since it affects the extraction rate and the extraction time at which equilibriums can be reached between the sample matrix and the sample headspace, as well as between the sample headspace and the stationary phase of the fiber. It is worth noting that increasing the temperature is not clearly associated with the extraction yield. A high extraction temperature does not provide better isolation and enrichment of volatile and semi-volatile compounds compared to lower temperatures. Furthermore, an increased temperature often adversely affects desorption of already adsorbed compounds at SPME fiber and incudes the adsorption of analytes back onto the polymeric stationary phase (Chmiel et al., 2017).

In order to find the best temperature range, the effect of temperature on the extraction yield of analytes was examined. Figure 2D shows the results of extraction at four different extraction temperatures. Eucalyptol, $\beta$-citral, and $\alpha$-citral were observed to have higher extraction yields at $40^{\circ} \mathrm{C}$ and $50^{\circ} \mathrm{C}$. For eucalyptol, $\alpha$-terpineol, $\beta$-citral, and $\alpha$-citral, the extraction efficiency could not reach the highest level even at $60{ }^{\circ} \mathrm{C}$. For most volatile compounds, the extraction yield was reduced at temperatures above $50{ }^{\circ} \mathrm{C}$. The reduced yield could be due to desorption of analytes from the fiber as a result of higher temperatures or formation of artifacts caused by thermal degradation. Previously, studies have proved that the temperature for extracting volatile compounds including terpenoids from food matrices shall not exceed $50^{\circ} \mathrm{C}$ due to possibilities of Millard reaction and Stecker degradation (Rocha et al., 2007; Williams et al., 2005). Therefore, the extraction temperature were decided to be $30^{\circ} \mathrm{C}$ and $50^{\circ} \mathrm{C}$ for further optimization by multivariate experimental designs.

\subsection{Response surface optimization of crucial HS-SPME conditions}

\section{RSM experiments and model fitting}

Based on results shown in Figure 1, the most important volatile components in LM fruit are $\alpha$-citral and $\beta$-citral (accounting for nearly $70 \%$ of the total volatile oil), which is in agreement with a previous report (Chen et al., 1984). Therefore, in this study, the peak area ratios of $\alpha$-citral and $\beta$-citral to the internal standard (4-methyl-2-pentanol) were selected as the response. The experimental design and peak area ratios of $\alpha$-citral and $\beta$-citra to the internal standard as the response are shown in
Table 2. As can be seen from Table 2, the peak area ratios of $\alpha$-citral and $\beta$-citra to the internal standard were ranging from $25.69 \pm 4.66$ to $67.86 \pm 10.58$.

The experimental data were fitted to a second-order polynomial model, and the regression of the obtained equation was evaluated from ANOVA. Additionally, the significance of the terms of the model was evaluated by $F$ test at $95 \%$ confidence level ( $p$ value). According to the results (Table 3), the terms $\mathrm{X}_{1}$ and $\mathrm{X}_{1}^{2}$ were considered significant. The Residual error of the model might be derived from random errors in the experimental process. The effect of different factors on the response value was in the order as follows: extraction temperature $(\mathrm{X} 1)>$ equilibrium time (X3) > extraction time (X2). The extraction temperature $(p=0.0363<0.05)$ had a significant effect on the extraction efficiency of terpenoids. After the exclusion of insignificant terms, Equation 2 could be written with real values as shown below:

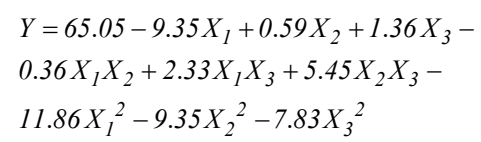

\section{Influence of different factors}

Three-dimensional graphs of the response surface (Figure 3) were obtained using Equation 2, illustrating the correlations between three independent variables and the response $(Y)$. Each three-dimensional graph reflects the response surface as a function of two factors, keeping the third factor at its central point. According to Equation 2, the linear term of extraction temperature $\left(\mathrm{X}_{1}\right)$ showed the highest coefficient value.

The effects of extraction temperature on the peak area ratios of $\alpha$-citral and $\beta$-citral to the internal standard are shown in Figure $3 \mathrm{~A}$ and $\mathrm{C}$, which were plots of response surface

Table 2. BBD for HS-SPME from LM immature fruits and the total peak area ratio of $\alpha$-citral and $\beta$-citral to the internal standard (4-methyl-2-pentanol).

\begin{tabular}{cllll}
\hline \multirow{2}{*}{ Test runs } & \multicolumn{3}{c}{ Independent variables } & $\begin{array}{c}\text { Dependent responses } \\
\text { (Peak area ratio) }\end{array}$ \\
\cline { 2 - 4 } & $\mathrm{X}_{1}\left({ }^{\circ} \mathrm{C}\right)$ & $\mathrm{X}_{2}(\mathrm{~min})$ & $\mathrm{X}_{3}(\mathrm{~min})$ & \\
\hline 1 & $30(-1)$ & $20(-1)$ & $20(0)$ & $60.90 \pm 10.28$ \\
2 & $50(1)$ & $20(-1)$ & $20(0)$ & $25.69 \pm 4.66$ \\
3 & $30(-1)$ & $50(1)$ & $20(0)$ & $62.71 \pm 4.49$ \\
4 & $50(1)$ & $50(1)$ & $20(0)$ & $26.04 \pm 5.90$ \\
5 & $30(-1)$ & $35(0)$ & $10(-1)$ & $51.10 \pm 7.07$ \\
6 & $50(1)$ & $35(0)$ & $10(-1)$ & $44.96 \pm 6.87$ \\
7 & $30(-1)$ & $35(0)$ & $30(1)$ & $41.09 \pm 5.63$ \\
8 & $50(1)$ & $35(0)$ & $30(1)$ & $44.29 \pm 3.66$ \\
9 & $40(0)$ & $20(-1)$ & $10(-1)$ & $47.29 \pm 7.27$ \\
10 & $40(0)$ & $50(1)$ & $10(-1)$ & $37.65 \pm 9.30$ \\
11 & $40(0)$ & $20(-1)$ & $30(1)$ & $47.18 \pm 7.56$ \\
12 & $40(0)$ & $50(1)$ & $30(1)$ & $59.35 \pm 3.37$ \\
13 & $40(0)$ & $35(0)$ & $20(0)$ & $64.67 \pm 10.76$ \\
14 & $40(0)$ & $35(0)$ & $20(0)$ & $64.27 \pm 7.84$ \\
15 & $40(0)$ & $35(0)$ & $20(0)$ & $67.86 \pm 10.58$ \\
16 & $40(0)$ & $35(0)$ & $20(0)$ & $64.10 \pm 1.28$ \\
17 & $40(0)$ & $35(0)$ & $20(0)$ & $64.33 \pm 7.73$ \\
\hline
\end{tabular}


Table 3. NOVA for a quadratic model of response surface.

\begin{tabular}{|c|c|c|c|c|c|}
\hline Factors & Sums of square & Degrees of freedom & mean square & $F$ value & $p$ value \\
\hline Model & 2214.46 & 9 & 246.05 & 2.35 & 0.1370 \\
\hline $\mathrm{X}_{1}$ & 699.75 & 1 & 699.75 & 6.67 & 0.0363 \\
\hline $\mathrm{X}_{2}$ & 2.75 & 1 & 2.75 & 0.026 & 0.8760 \\
\hline $\mathrm{X}_{3}$ & 14.88 & 1 & 14.88 & 0.14 & 0.7176 \\
\hline $\mathrm{X}_{1} \mathrm{X}_{2}$ & 0.53 & 1 & 0.53 & 0.005 & 0.9452 \\
\hline $\mathrm{X}_{1} \mathrm{X}_{3}$ & 21.81 & 1 & 21.81 & 0.21 & 0.6622 \\
\hline $\mathrm{X}_{2} \mathrm{X}_{3}$ & 118.92 & 1 & 118.92 & 1.13 & 0.3224 \\
\hline $\mathrm{X}_{1}^{2}$ & 592.18 & 1 & 592.18 & 5.64 & 0.0492 \\
\hline $\mathrm{X}_{2}^{2}$ & 368.23 & 1 & 368.23 & 3.51 & 0.1031 \\
\hline $\mathrm{X}_{3}^{2}$ & 257.93 & 1 & 257.93 & 2.46 & 0.1609 \\
\hline Residual error & 734.35 & 7 & 104.91 & - & - \\
\hline Lack of fit & 724.28 & 3 & 241.43 & 95.90 & 0.0004 \\
\hline Net errors & 10.07 & 4 & 2.52 & - & - \\
\hline Cor total & 2948.81 & 16 & - & - & - \\
\hline
\end{tabular}

$p<0.05$, significant; $p<0.01$, highly significant. The $\mathrm{F}$ value indicates the influence of each factor on the evaluation index and the greater the $\mathrm{F}$ value, the greater the impact on the index.

A

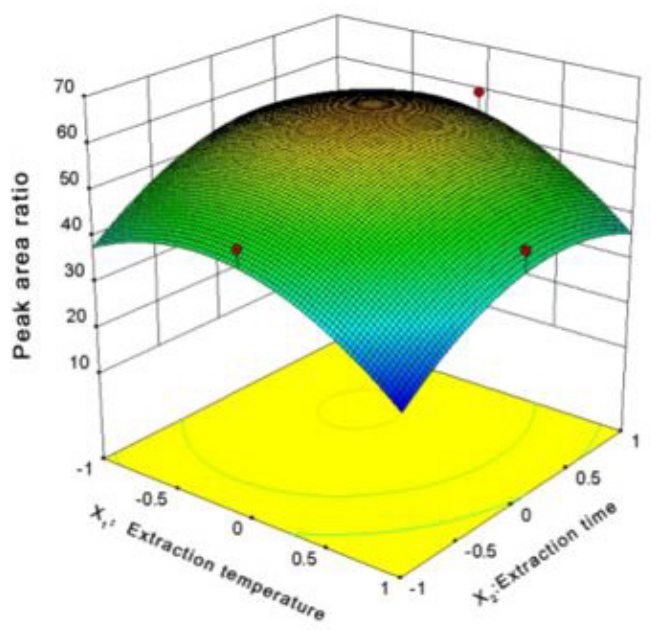

B

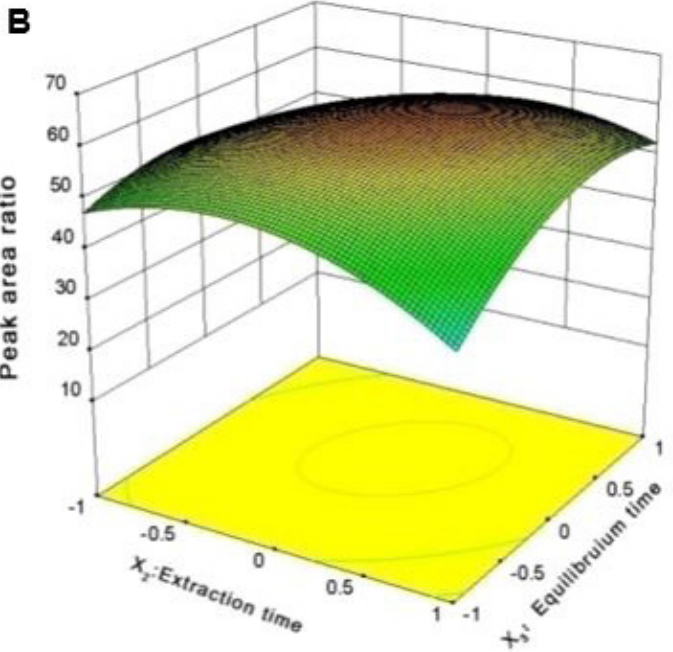

C

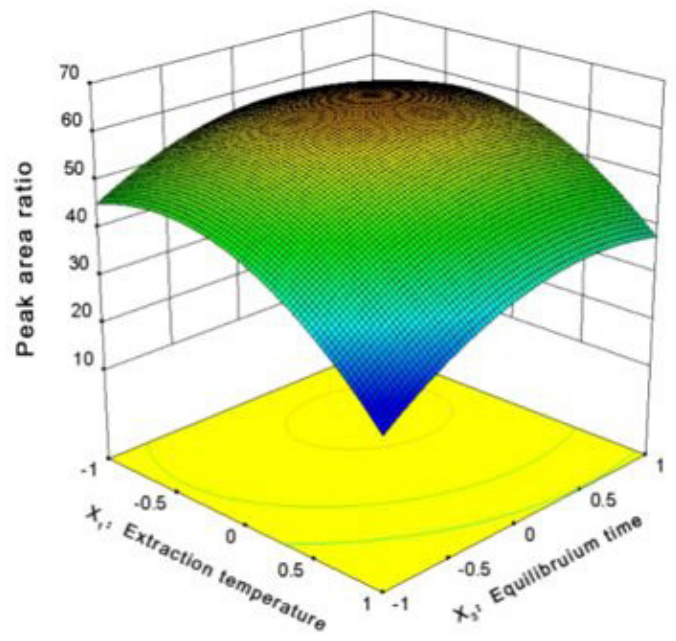

Figure 3. Response surface plots of extraction yields of $\alpha$-citral and $\beta$-citral (Y1) as a function of: (A) extraction temperature and time at a constant equilibrium time of $20 \mathrm{~min}$; (B) duration of extraction and equilibrium steps at a constant temperature of $40^{\circ} \mathrm{C}$; and (C) extraction temperature and equilibrium time at a fixed extraction time of $35 \mathrm{~min}$. 
as a function of extraction temperature and extraction time (Figure 3A), and extraction temperature and equilibrium time (Figure 3C), respectively. The peak area ratios of $\alpha$-citral and $\beta$-citral increased with the increase of extraction temperature. However, when the extraction temperature increased to a certain extent, the peak area ratio tended to decrease. The extraction temperature had a significant impact on the peak area ratios of $\alpha$-citral and $\beta$-citral $(p=0.0363)$, and equilibrium time also had a notable influence on the peak area ratios of $\alpha$-citral and $\beta$-citral $(p=0.7176)$. The increase of both factors accelerated extraction of $\alpha$-citral and $\beta$-citral.

The extraction time and equilibrium time did not significantly affect the peak area ratio as indicated by the gentle slope of interaction response surface shown in Figure 3B. This result indicated that the extraction time was not a significant factor, agreeing with the low coefficient in Equation 2 and the data shown in Table 3, in which the linear term of extraction time showed lower $F$ values.

The extraction conditions were optimized to maximize the extraction of terpenoids from LMIF. Through data processing and analysis of the response surface graphs, the optimal HS-SPME conditions were determined as follows: an extraction and equilibrium temperature of $46.1{ }^{\circ} \mathrm{C}$, an extraction time of $35.80 \mathrm{~min}$, and an equilibrium time of $20.48 \mathrm{~min}$, with a desirability value of 0.978 . Under these conditions, the extraction ratio of $\alpha$-citral and $\beta$-citral was predicted by RSM models to be 66.92 . Further, considering the equipment operating parameters, the optimal extraction conditions could be modified as follows: an extraction and equilibrium temperature of $46^{\circ} \mathrm{C}$, an extraction time of $36 \mathrm{~min}$, and an equilibrium time of $20 \mathrm{~min}$. Under the modified conditions, the experimental values of the $\alpha$-citral and $\beta$-citral ratio was $65.78 \pm 5.72$ (from three parallel experiments). The predicted values agreed well with experimental values, verifying the validity of the designed models with satisfactory repeatability.

\section{Conclusions}

As a spice or appetizing cold dish, aroma is a very important aspect of quality for LMIF. However, there exists no prior reliable methods for extracting aroma components from LMIF. This study established the optimal conditions for using HS-SPME to extract aroma components, terpenoids in particular, from LMIF by conducting a series of single factor-experiments and analyzing response surface graphs. The optimal parameters are: 50/30 $\mu \mathrm{m}$ of DVB/CAR/PDMS SPME fiber, $2.0 \mathrm{~g}$ of added sodium chloride, desorption at a temperature of $250^{\circ} \mathrm{C}$ for $3 \mathrm{~min}$, extraction and equilibrium temperature of $46^{\circ} \mathrm{C}$. Using these parameters, the predicted value of extraction was 66.92 , while the experimental value was $65.78 \pm 5.72$. The prediction value matched well with the experimental value with good repeatability, indicating that the designed model was valid. Our findings provide a valuable clue for extracting aroma compounds, especially terpenoids, from LMIF.

\section{Acknowledgements}

This work was fnancially supported by the State Key Research and Development Plan "Modern Food Processing and Food Storage and Transportation Technology and
Equipment" (No. 2018YFD0400104) and by the Beijing Advanced Innovation Center for Food Nutrition and Human Health (No. 20171010).

\section{References}

Bezerra, M. A., Santelli, R. E., Oliveira, E. P., Villar, L. S., \& Escaleira, L. A. (2008). Response surface methodology (RSM) as a tool for optimization in analytical chemistry. Talanta, 76(5), 965-977. http:// dx.doi.org/10.1016/j.talanta.2008.05.019. PMid:18761143.

Chen, H. P., Yi, H. X., Liu, Y. Q., \& Zhang, S. Y. (1984). Chemical composition of the essential oil from Litsea mollis Hemsl. fruits. Chinese Traditional and Herbal Drugs, 15(11), 13-15.

Chen, W., Wang, W. P., Zhang, H. S., \& Huang, Q. (2012). Optimization of ultrasonic-assisted extraction of water-soluble polysaccharides from Boletus edulis mycelia using response surface methodology. Carbohydrate Polymers, 87(1), 614-619. http://dx.doi.org/10.1016/j. carbpol.2011.08.029.

Chinese Academy of Sciences. (2019). Flora of China. Retrieved from http://www.iplant.cn/info/Litsea\%20mollis?t=foc

Chmiel, T., Kupska, M., Wardencki, W., \& Namieśnik, J. (2017). Application of response surface methodology to optimize solid-phase microextraction procedure for chromatographic determination of aroma-active monoterpenes in berries. Food Chemistry, 221, 1041-1056. http://dx.doi.org/10.1016/j.foodchem.2016.11.057. PMid:27979057.

Klesk, K., Qian, M., \& Martin, R. R. (2004). Aroma extract dilution analysis of cv. Meeker (Rubus idaeus L.) red raspberries from Oregon and Washington. Journal of Agricultural and Food Chemistry, 52(16), 5155-5161. http://dx.doi.org/10.1021/jf0498721. PMid:15291490.

Ma, Q. L., Hamid, N., Bekhit, A. E. D., Robertson, J., \& Law, T. F. (2013). Optimization of headspace solid phase microextraction (HS-SPME) for gas chromatography mass spectrometry (GC-MS) analysis of aroma compounds in cooked beef using response surface methodology. Microchemical Journal, 111, 16-24. http://dx.doi. org/10.1016/j.microc.2012.10.007.

Martins, A. C., Bukman, L., Vargas, A. M. M., Barizão, É. O., Moraes, J. C. G., Visentainer, J. V., \& Almeida, V. C. (2013). The antioxidant activity of teas measured by the FRAP method adapted to the FIA system: optimising the conditions using the response surface methodology. Food Chemistry, 138(1), 574-580. http://dx.doi. org/10.1016/j.foodchem.2012.10.143. PMid:23265526.

National Institute of Standards and Technology - NIST. (2019). Retrieved from http://webbook.nist.gov/chemistry

Peña, R. M., Barciela, J., Herrero, C., \& García-Martín, S. (2005). Optimization of solidphase microextraction methods for GC-MS determination of terpenes in wine. Journal of the Science of Food and Agriculture, 85(7), 1227-1234. http://dx.doi.org/10.1002/jsfa.2121.

Rocha, S. M., Coelho, E., Zrostlíková, J., Delgadillo, I., \& Coimbra, M. A. (2007). Comprehensive two-dimensional gas chromatography with time-of-flight mass spectrometry of monoterpenoids as a powerful tool for grape origin traceability. Journal of Chromatography. A, 1161(1-2), 292-299. http://dx.doi.org/10.1016/j.chroma.2007.05.093. PMid:17585921.

Vichi, S., Guadayol, J. M., Caixach, J., López-Tamames, E., \& Buxaderas, S. (2006). Monoterpene and sesquiterpene hydrocarbons of virgin olive oil by headspace solid-phase microextraction coupled to gas chromatography/mass spectrometry. Journal of Chromatography A, 1125(1), 117-123. http://dx.doi.org/10.1016/j.chroma.2006.05.029. PMid:16756984. 
Williams, A., Ryan, D., Olarte Guasca, A., Marriott, P., \& Pang, E. (2005). Analysis of strawberry volatiles using comprehensive two-dimensional gas chromatography with headspace solid-phase microextraction. Journal of Chromatography. B, Analytical Technologies in the Biomedical and Life Sciences, 817(1), 97-107. http://dx.doi. org/10.1016/j.jchromb.2004.05.021. PMid:15680793.

Xiao, Y., \& Li, L. (2007). Research advance on essential oils from Litsea and their biological activity. Yunnan Chemical Technology, 34(5), 85-92.
Yang, Y. N., Zheng, F. P., Yu, A. N., \& Sun, B. G. (2019). Changes of the free and bound volatile compounds in Rubus corchorifolius L. f. fruit during ripening. Food Chemistry, 287, 232-240. http://dx.doi. org/10.1016/j.foodchem.2019.02.080. PMid:30857694.

Zhang, X. (2015). Spices and food culture in native minorities of Dehong. Agricultural Science and Technology, 16(2), 380-383.

Zhou, T. D. (1995). Studies on antibechic and expectorant effects and chemical components of volatile oil from Litsea mollifolia chun flores. Chinese Journal of Modern Applied Pharmacy, 12(3), 16-17. 